Revue internationale P.M.E.

Économie et gestion de la petite et moyenne entreprise

\title{
La coentreprise comme lien de collaboration entre les PME des pays développés et en voie de développement
}

\section{Rick Donckels et Johan Lambrecht}

Volume 8, numéro 1, 1995

URI : https://id.erudit.org/iderudit/1008273ar

DOI : https://doi.org/10.7202/1008273ar

Aller au sommaire du numéro

Éditeur(s)

Presses de l'Université du Québec

ISSN

0776-5436 (imprimé)

1918-9699 (numérique)

Découvrir la revue

Citer cet article

Donckels, R. \& Lambrecht, J. (1995). La coentreprise comme lien de collaboration entre les PME des pays développés et en voie de développement. Revue internationale P.M.E., 8(1), 9-29. https://doi.org/10.7202/1008273ar
Résumé de l'article

Les études empiriques sur la coentreprise dans un pays en voie de développement, où les PME d'un pays développé et du tiers monde tiennent les rôles principaux, sont très limitées. Dans cet article, nous essayons de combler cette lacune. D’abord, nous avons établi un cadre de référence dans lequel nous démontrons que la coentreprise profite autant aux entrepreneurs et PME des pays développés qu'à ceux et celles des pays en voie de développement. Nous avons ensuite formulé un certain nombre d'hypothèses, que nous avons testées en deux étapes analytiques. Enfin, nous terminons en formulant des recommandations à l'adresse des PME et de l'infrastructure d'appui. 


\title{
La coentreprise comme lien de collaboration entre les PME des pays développés et en voie de développement
}

\author{
Rik DONCKELS* \\ Johan LAMBRECHT** \\ Centre d'études des PME \\ K.U. Brussel
}

MOTS CLÉS

Coentreprise - PME - Pays développés -

Pays en voie de développement - Levier de développement

\begin{abstract}
RÉSUMÉ
Les études empiriques sur la coentreprise dans un pays en voie de développement, où les PME d'un pays développé et du tiers monde tiennent les rôles principaux, sont très limitées. Dans cet article, nous essayons de combler cette lacune. D'abord, nous avons établi un cadre de référence dans lequel nous démontrons que la coentreprise profite autant aux entrepreneurs et PME des pays développés qu'à ceux et celles des pays en voie de développement. Nous avons ensuite formulé un certain nombre d'hypothèses, que nous avons testées en deux étapes analytiques. Enfin, nous terminons en formulant des recommandations à l'adresse des PME et de l'infrastructure d'appui.
\end{abstract}

* Rik Donckels est professeur à la K.U. Brussel et à la K.U. Leuven. Il est directeur du Centre d'études des PME à la K.U. Brussel. Il est titulaire d'une licence en sciences mathématiques et d'un Ph.D. en économie de la K.U. Leuven. M. Donckels est l'auteur de diverses études théoriques et empiriques sur le monde de la PME. Sa bibliographie comporte plusieurs monographies ainsi que de nombreuses contributions à des revues scientifiques tant belges qu'étrangères.

** Johan Lambrecht est titulaire d'un M.A. en économie de la K.U. Leuven et d'une maîtrise d'analyse quantitative en sciences sociales de la K.U. Brussel. Il travaille comme collaborateur scientifique au Centre d'études des PME de la K.U. Brussel. M. Lambrecht est l'auteur de plusieurs publications concernant les PME. Adresse : Centre d'études des PME, K.U. Brussel, Vrijheidslaan 17, 1080 Bruxelles, Belgique.

Les auteurs remercient l'Administration générale de la coopération au développement (AGCD) pour le financement de l'étude sur laquelle le présent article est basé. Ils tiennent également à remercier chaleureusement le professeur Léopold Bragard et Isabelle Leboulle du Centre de recherche et de documentation PME, Université de Liège, pour leur précieuse collaboration à l'enquête. 


\begin{abstract}
To date, there has been limited empirical research on joint ventures in developing countries between SMEs from industrial and Third World countries. With this article, we shall try to fill this gap. First, a framework in which we demonstrate that entrepreneurs and SMEs from both developed and developing countries benefit from a joint venture is presented. After that, a number of hypotheses are formulated and tested in two analytical steps. We conclude by formulating recommendations for the SMEs and supporting institutions.
\end{abstract}

\title{
RESUMEN
}

Hasta ahora los estudios empíricos en los países en vías de desarrollo sobre las joint-ventures entre les PyMEs de los países industrializados y del tercer mundo no eran muy numerosos. Con este artículo hemos intentado paliar este vacío. Hemos comenzado presentando un marco con el que demostrar que una joint-venture beneficia tanto a los empresarios como a las PyMEs de los países desarrollados o en vías de desarrollo. Después, hemos formulado y examinado una serie de hipótesis en dos etapas analíticas. Para terminar hemos propuesto una serie de recomendaciones para las PyMEs y las instituciones que las apoyan.

\section{Introduction}

Pour l'heure, nous sommes les témoins d'un nombre sans cesse croissant d'alliances entre des entreprises qui resserrent leurs liens de collaboration afin de renforcer leur position concurrentielle sur le marché intérieur et de développer ou d'étendre leurs activités internationales. La coentreprise est l'une de ces formes de collaboration qui a été étudiée en détail par les chercheurs. Toutefois, ces études ont rarement touché les PME. En outre, quand la coentreprise a lieu dans des pays en voie de développement et que les entrepreneurs de pays industrialisés et du tiers monde tiennent les rôles principaux, la littérature s'intéresse encore moins aux PME.

Dans cet article, nous essayons de combler cette lacune à partir d'une étude pilote et d'une enquête plus large. La coentreprise est ici définie comme une entreprise dans un pays en voie de développement (en Afrique, en Asie et en Amérique latine) dont le capital est partagé entre un entrepreneur d'un pays industrialisé et un autre d'un pays en voie de développement. Ces dirigeants de PME apportent donc une contribution au capital. Celle-ci peut, par exemple, consister en technologie, appuyée par la suite par des capitaux. Par cette participation, chaque entrepreneur acquiert un certain contrôle sur l'entreprise commune. 


\section{Vers un cadre de référence}

Pour élaborer le cadre de référence, nous avons utilisé la théorie et les résultats d'une étude pilote qui a été effectuée auprès de 70 témoins privilégiés de l'infrastructure d'appui aux PME en Belgique. Les entretiens avec ces personnes étaient axés sur les thèmes suivants : les obstacles au développement d'une alliance, les clés du succès d'une telle action, les dispositions complémentaires qui pourraient être prises pour améliorer la situation et l'importance de l'esprit d'entreprise pour les pays en voie de développement et le rôle que peuvent y jouer les dirigeants de PME belges. Nous avons rassemblé aux schémas 1 et 2 les observations concordantes tirées de la littérature et des entrevues.

Au schéma 1, on peut voir les entrepreneurs qui s'impliquent totalement dans leur entreprise. Leurs caractéristiques déterminent, bien sûr, l'évolution de leur entreprise et celle-ci influence à son tour l'entrepreneur. En outre, on peut constater l'interdépendance entre les dirigeants de PME et leur entreprise, d'une part, et l'environnement extérieur, d'autre part. En fait, les entrepreneurs et leur PME font face à des facteurs environnementaux pouvant contrecarrer leurs activités ou leur donner un coup de fouet. Entrepreneurs et PME peuvent également émettre des signaux et avoir de la sorte un impact sur le monde extérieur. Les sections concentriques hachurées indiquent que la collaboration rapproche les organes du pays industrialisé et du pays en voie de développement. Il ressort de ce schéma que des caractéristiques propres aux entrepreneurs et aux entreprises et l'environnement extérieur sont des facteurs cruciaux de la collaboration. En conséquence, nous analyserons ces éléments dans la partie empirique.

\section{SCHÉMA 1}

\section{Les éléments d'un lien de collaboration}

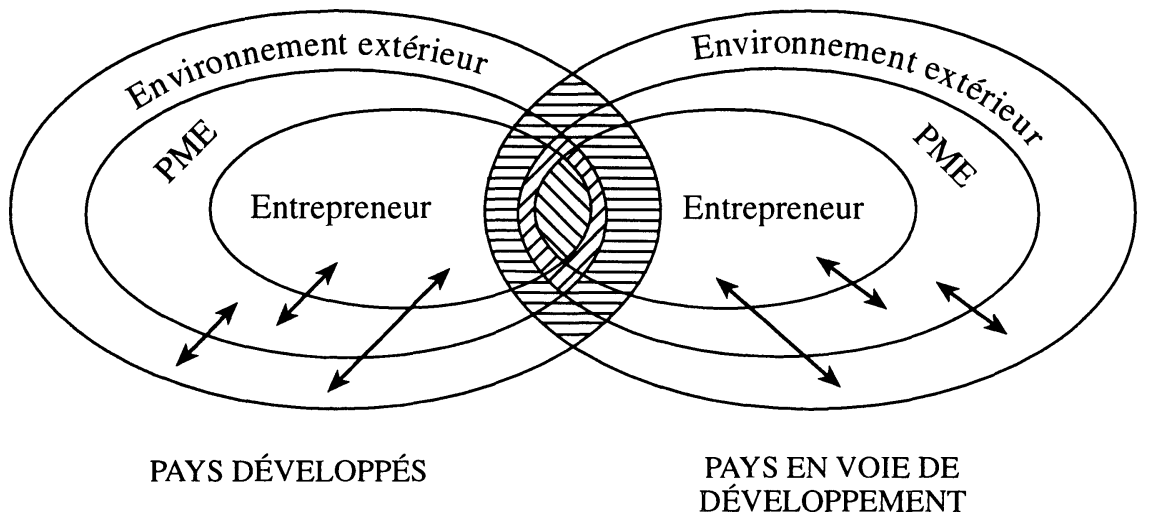

Revue internationale P.M.E., vol. 8, nº 1, 1995 
Pour sa part, le schéma 2 indique que les entrepreneurs constituent le cœur de toute collaboration. Ils se doivent donc de développer des mécanismes afin de faire concorder leurs objectifs. Nous démontrerons, ci-après, que la coentreprise peut répondre aux motivations de collaboration qu'ont les entrepreneurs et les PME du pays développé et du pays en voie de développement.

SCHÉmA 2

\section{Les raisons de la collaboration}

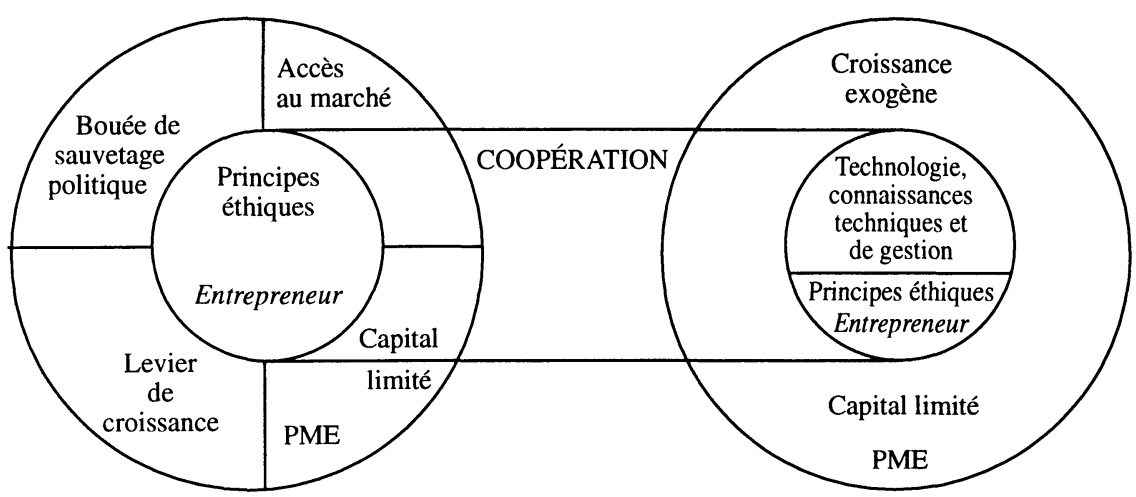

PAYS INDUSTRIALISÉS

PAYS EN VOIE DE

DÉVELOPPEMENT

\subsection{Les PME des pays industrialisés}

Certains dirigeants de PME des pays industrialisés peuvent se laisser guider par des principes éthiques. Même si les considérations d'affaires primeront toujours, le sens de leur responsabilité sociale peut aussi être source de collaboration avec un entrepreneur d'un pays en voie de développement. Ils veulent lui transférer de la technologie et des connaissances de gestion et contribuer ainsi à l'amélioration socio-économique de ce pays en voie de développement. On constate, dès lors, leur déception lorsque le transfert de connaissances s'arrête ou que des investissements sont mal ou pas utilisés du tout (Dagevos et De Groot, 1990). Buckley et Casson (1988) soulignent également que l'implication est plus importante lorsque l'éthique joue un rôle dans l'opération. Dawson (1982) insiste sur le fait que l'entrepreneur du pays industrialisé ressent une satisfaction morale lorsqu'il contribue à soulager les besoins des plus défavorisés dans ces pays. Une forme de collaboration dont le dirigeant de 
PME peut se réjouir d'un point de vue éthique est bien la coentreprise. En effet, on voit rarement les partenaires mettre autant leurs forces et leurs moyens à contribution que dans cette association (Harrigan, 1988). Pour Kogut (1988), les coentreprises constituent un véhicule idéal pour échanger informations et technologies et pour obtenir de meilleurs résultats. D'après lui, il s'agit d'une conséquence de la « prise d'otage réciproque » résultant de l'implication commune dans les actifs et de la répartition des frais et bénéfices. En d'autres mots, la coentreprise offre de bonnes perspectives à long terme et présente, par conséquent, de fortes chances d'obtenir unestabilité dans la coopération.

Comme premier avantage d'une telle collaboration pour les PME d'un pays industrialisé, il y a l'accès au marché. Pour celles-ci, il est particulièrement difficile de pénétrer les marchés des pays en voie de développement. Il faut, en effet, franchir les barrières non seulement géographiques mais également psychologiques; il peut donc être préférable de s'adresser à un partenaire local. Ce dernier possède une meilleure vision de la concurrence, du comportement des consommateurs, de la situation dans le secteur et sur le plan de la distribution (Schillaci, 1987). En outre, un entrepreneur local constitue un excellent guide lorsqu'il s'agit d'affronter la jungle administrative qui règne souvent dans les pays en voie de développement. Mais ici se pose la question de savoir pourquoi une coentreprise constitue une meilleure porte d'entrée que d'autres liens de collaboration. Les licences, par exemple, permettent une représentation rapide sur le marché avec un investissement relativement limité (Lei et Slocum, 1991). Il existe, néanmoins, des connaissances spécifiques, telles que celles ayant trait aux caractéristiques du pays, qui ne sont pratiquement transmissibles que par l'intermédiaire d'une coentreprise (Hennart, 1988). C'est sûrement le cas lorsqu'il existe de grandes différences culturelles entre les pays des deux partenaires. Ainsi, Kogut et Singh (Kogut, 1988) ont remarqué que les coentreprises sont plus fréquentes lorsque la distance culturelle est grande.

Un capital limité est le deuxième ressort de la collaboration. La plupart des PME ne disposent ni de grands moyens financiers, ni de ressources organisationnelles suffisantes pour installer de leur propre initiative une filiale commerciale, et encore moins d'une unité de production complète, dans un pays en voie de développement (Berlew, 1984). Une coentreprise peut offrir, par conséquent, un ballon d'oxygène à la PME du pays industrialisé, car le capital est partagé avec l'entrepreneur local. Cependant, pour cette forme de collaboration, elle devra disposer d'une bonne réserve financière.

Samuels, Greenfield et Mpuku (1992) ont remarqué que les PME des West-Midlands (Angleterre) ont enregistré de bons résultats avec les pays en 
voie de développement. Un cinquième des firmes interrogées ont les pays en voie de développement comme principal marché d'exportation ; $37 \%$ et $21 \%$ ont placé respectivement l'Union européenne et l'Amérique du Nord en première position. On peut donc en déduire qu'une relation économique avec un pays en voie de développement peut être un instrument de croissance très utile. En résumé, la coentreprise est une voie possible pour les PME qui recherchent la croissance, car cette forme d'alliance permet de pénétrer un marché par l'intérieur. De même, l'accroissement de capacité, l'intégration verticale ou la diversification font de la coentreprise une source de croissance (Schillaci, 1987).

Les entrepreneurs actifs dans les pays en voie de développement doivent tenir compte du fait qu'ils peuvent naviguer en eaux politiques troubles. Dans une mer sujette à autant de tourbillons politiques, un partenaire local peut faire office de bouée de sauvetage. Grâce à son influence politique, il peut éviter que ces brusques interventions gouvernementales portent atteinte à ses actions et à celles de son associé (Contractor et Lorange, 1988). Toutefois, la prudence est de rigueur avec un partenaire qui se laisse trop porter par le courant politique existant. De plus, l'apport financier de l'entrepreneur local peut être une protection contre les «nationalisations » de la part des autorités du pays en voie de développement. Souvent, d'ailleurs, celles-ci exigent une participation locale, ce qui peut constituer une garantie supplémentaire pour la coentreprise.

\subsection{Les PME des pays en voie de développement}

Du côté des entrepreneurs du pays en voie de développement, ceux-ci peuvent acquérir, grâce à la collaboration, de la technologie et des connaissances techniques et de gestion. Par définition, ces connaissances sont intimement liées à celui qui les possède et leur échange, par conséquent, ne peut que s'appuyer sur des contacts humains étroits (Hennart, 1988). Ces derniers sont présents en surabondance dans les coentreprises de PME où la collaboration a moins lieu entre entreprises qu'entre entrepreneurs. Pour le dirigeant de PME du pays en voie de développement, la coentreprise représente donc une manière efficace d'apprendre. Il doit cependant y être disposé et en avoir les capacités, et pour cela, lui et ses partenaires doivent jouer franc jeu (Hamel, 1991).

Les principes éthiques peuvent également être présents chez l'entrepreneur local. Celui-ci peut rechercher cette collaboration, recevoir de la technologie et des connaissances techniques et de gestion, notamment, pour renforcer son pays dans le domaine socio-économique. Il existe une forte probabilité pour qu'une coentreprise avec une PME d'un pays industrialisé contribue à ce résultat. La coentreprise est, en effet, une bonne garantie de stabilité. De plus, les PME travaillent sur des niches et peuvent donc mieux répondre à des 
besoins locaux. Dès lors, il est concevable que l'entrepreneur local ne sera pas considéré comme un mal nécessaire, mais comme un joueur indispensable. Dans le cadre d'une stratégie économique globale, par contre, il est possible que l'entrepreneur local serve uniquement d'alibi politique, surtout lorsque l'économie du pays en voie de développement est impliquée dans une production mondiale contrôlée par des multinationales. Dans ce cas, un contrôle strict de l'entrepreneur local est nécessaire et un partenaire rétif peut vite devenir un boulet (Harrigan, 1988 ; Gomes-Casseres, 1989). Les multinationales qui conservent une coentreprise avec de piètres résultats dans un pays en voie de développement le font souvent pour des raisons politiques (Beamish, 1987). La PME de pays industrialisés, en raison de son important investissement en temps et en argent, mettra tout en œuvre afin d'assurer la réussite de la coentreprise dans le pays en voie de développement, alors que les multinationales, en raison de leurs opérations à l'échelle mondiale, n'insuffleront probablement pas d'air nouveau dans des filiales à l'agonie (Simiar, 1983).

Pour la PME de pays en voie de développement, la coentreprise constitue également un bon moyen pour réaliser ses objectifs de croissance et de recherche de capitaux. Grâce à l'obtention d'équipements techniques et de gestion plus développés, la PME locale sera mieux armée non seulement sur le plan local, mais également sur le plan international : elle pourra mieux se défendre (Datta, 1988). En outre, elle surmontera plus facilement les difficultés de croissance, car ces dernières sont de nature exogène. Il existera, en effet, un partenaire pour soutenir une situation financière précaire.

\section{Hypothèses}

Nos hypothèses ont été établies à partir du point de vue des entrepreneurs des pays industrialisés, mais elles n'en tiennent pas moins compte des pays en voie de développement et de leurs dirigeants de PME. Elles se situent dans le prolongement du cadre de référence et reposent donc sur la littérature et l'étude pilote.

\subsection{Transactions préalables}

Au schéma 1, nous avons vu que les liens de collaboration supposent l'interpénétration entre la direction, l'entreprise et son environnement. L'obtention d'une cohérence n'est pas du tout facile, a fortiori quand il s'agit de la création d'une coentreprise dans un pays en voie de développement. Les partenaires doivent, en effet, réaliser un creuset d'une culture d'entreprise traditionnelle et non traditionnelle dans des liens formels. En conséquence, on peut s'attendre à ce qu'ils concluent d'abord une alliance moins étroite, au 
cours de laquelle ils verront s'ils peuvent accorder leurs violons. Si tel est le cas, ils pourront éventuellement poursuivre l'expérience de la coentreprise.

Une étude de l'Organisation des Nations Unies pour le développement industriel en France révèle que la majorité des entreprises françaises souhaitent d'abord vendre leurs produits avant de considérer les possibilités d'une collaboration (Pasquet, 1988). En outre, des liens de collaboration moins étroits subsistent souvent parallèlement à la coentreprise.

Hypothèse 1 : Une coentreprise dans un pays en voie de développement est le plus souvent précédée par l'établissement de liens de collaboration moins étroits et accompagnée d'autres formes de liens.

\subsection{La recherche d'informations}

Étant donné que l'établissement d'un lien de collaboration avec un entrepreneur d'une autre culture n'a rien d'une sinécure, on peut supposer que l'entrepreneur du pays industrialisé qui établit une coentreprise dans un pays en voie de développement devra réunir de nombreuses informations avant de conclure une telle coentreprise. Selon les intervenants auprès des PME que nous avons interrogés, découvrir et analyser les sources de renseignements dans son propre pays constitue la première tâche de l'entrepreneur du pays industrialisé. Ensuite, il est indispensable d'examiner attentivement les aspects économiques, sociaux, politiques et culturels du pays en voie de développement concerné (Datta, 1988).

Hypothèse 2: La constitution d'une coentreprise dans un pays en voie de développement va inévitablement de pair avec une recherche approfondie d'informations.

\subsection{Les difficultés plus importantes}

Une coentreprise dans un pays en voie de développement représente un accord difficile et fragile (Newman, 1992). Il est, par conséquent, plausible que les personnes désirant mettre une coentreprise sur pied dans ces pays rencontrent plus de difficultés que ceux qui concluent des alliances moins poussées.

Hypothèse 3: Les entrepreneurs qui établissent des liens de coentreprise dans un pays en voie de développement font face à plus de difficultés que ceux qui concluent des accords moins étroits. 


\subsection{Le besoin d'un plan d'affaires écrit et détaillé}

Ferdows (1980), en étudiant les investissements en Iran de 40 PME de pays industrialisés, a constaté que les entrepreneurs dont le projet a été couronné de succès n'avaient pas suivi un plan d'affaires écrit. Ferdows attribue cette absence de planification précise à l'incertitude de l'environnement, à la nouveauté et à la dépendance réciproque des activités.

Hypothèse 4: Un plan d'affaires écrit détaillé n'est pas considéré comme déterminant pour la réussite d'une coentreprise dans un pays en voie de développement.

\subsection{L'exportation comme stimulant}

Dagevos et De Groot (1990) ont remarqué, en étudiant l'économie de la province du Brabant aux Pays-Bas, que l'exportation constitue la façon la plus utilisée pour travailler avec les pays en voie de développement. Comme nous l'avons mentionné dans le cadre de référence, les pays en voie de développement peuvent constituer un marché d'exportation important pour des PME. Nous supposons que la coentreprise vient souvent compléter un lien de collaboration moins étroit (voir l'hypothèse 1) et que les PME qui mettent sur pied une coentreprise dans un pays en voie de développement sont plutôt celles qui y réalisent déjà un bon chiffre d'affaires. Leur bonne relation commerciale prouve qu'il existe un marché pour leurs produits ainsi que des possibilités de collaboration étroite. Il est donc plausible que ces PME conçoivent la coentreprise comme la suite logique des liens distendus.

Hypothèse 5: Les PME qui entrent dans une coentreprise dans un pays en voie de développement sont plutôt celles qui y réalisent déjà un bon chiffre d'affaires.

\subsection{Les caractéristiques propres aux entrepreneurs}

L'enquête sur l'internationalisation des PME belges a montré que les chefs d'entreprise actifs sur le plan international connaissent un nombre bien plus important de langues (Donckels et Aerts, 1993). De plus, les dirigeants de PME belges étudiés sont d'autant plus actifs dans leurs relations avec un pays en voie de développement que leur degré de scolarisation et de connaissance des langues est élevé (Lambrecht, 1993). Une étude réalisée en Belgique et portant sur les dirigeants de PME et leur environnement a clairement indiqué que la position dans le réseau d'affaires national ou international se renforce proportionnellement au degré de scolarisation (Donckels et Elshout, 1992). 
Nous pouvons ainsi conclure que le degré de scolarisation et de connaissance des langues joue un rôle crucial pour les coentreprises dans les pays en voie de développement.

Hypothèse 6: Le degré de scolarisation et de connaissance des langues influe sur le processus décisionnel d'une coentreprise dans un pays en voie de développement.

\section{Méthodologie}

\subsection{La collecte des données}

Comme nous l'avons mentionné, l'enquête a débuté en 1992 par l'étude approfondie de la littérature et l'interview de 70 témoins privilégiés d'organismes belges d'appui aux PME. Ces deux sources d'information ont livré suffisamment d'éléments pour permettre d'amorcer l'enquête auprès des entrepreneurs. Cinq cents dirigeants de PME belges qui entretiennent des relations d'affaires avec un pays en voie de développement ont été choisis au hasard, sur la base de catalogues et de banques de données ; ils ont été interrogés par téléphone par un bureau de marketing spécialisé. Notons qu'une PME belge est une entreprise comptant moins de 100 employés et que sont considérés comme pays en voie de développement les pays à faibles et moyens revenus d'Afrique, d'Asie et d'Amérique latine : leur PNB n'a pas atteint 2450 \$ US par personne en 1989 (critère utilisé par la Banque mondiale). En outre, avant l'enquête téléphonique, les entrepreneurs avaient reçu une lettre de présentation ainsi qu'une liste de pays en voie de développement. Finalement, 480 enquêtes ont été validées.

\subsection{Le traitement des données}

Les résultats de l'enquête téléphonique sont présentés ici de façon descriptive et analytique. Dans la partie descriptive, on présente des fréquences relatives. Les hypothèses ont été testées en deux étapes analytiques. Dans un premier temps, les enquêtes partielles ont été comparées par des tests chi-carré, ce qui a permis de rechercher les différences significatives existant entre, d'une part, les entrepreneurs qui ont mis ou désirent mettre sur pied une coentreprise dans un pays en voie de développement (entrepreneurs «pro-coentreprise ») et, d'autre part, ceux qui ne recherchent pas ce type d'alliance (entrepreneurs «anti-coentreprise»). Les résultats ont ensuite été affinés en faisant appel à l'analyse non linéaire en composantes principales. Nous avons utilisé cette 
technique - réalisée à l'aide du programme informatique Princals ${ }^{1}$ - parce que les variables à analyser se situaient à tous les niveaux de mesure (intervalle, ordinal et nominal).

\section{Analyse empirique}

Dans cette section, nous avons établi le profil des 89 entrepreneurs interrogés qui ont conclu ou veulent mettre sur pied une coentreprise dans un pays en voie de développement. Leur profil est comparé à celui des 385 dirigeants de PME qui excluent cette forme de collaboration. Nous commençons par montrer les différences significatives en comparant les résultats des deux enquêtes partielles (la valeur $\mathrm{p}$ est indiquée entre parenthèses). En outre, les variables individuelles ont été rassemblées sous quatre dénominateurs: schéma relationnel avec les pays en voie de développement, travail d'exploration, expériences et caractéristiques propres aux entrepreneurs. Ensuite, nous les soumettons à l'analyse non linéaire en composantes principales.

\subsection{Les comparaisons des enquêtes partielles}

\subsubsection{Le schéma relationnel avec les pays en voie de développement}

Le graphique de la page suivante représente les types de relations des entrepreneurs qui ont conclu ou veulent mettre sur pied une coentreprise dans un pays en voie de développement et ceux qui excluent cette forme de coopération. Il faut souligner que tous les dirigeants de PME engagés maintenant ou dans le futur dans une coentreprise possèdent d'autres liens économiques avec des pays en voie de développement. En outre, $72 \%$ des entrepreneurs qui participent actuellement à une coentreprise ont commencé par une forme de collaboration moins étroite. L'hypothèse 1 est donc confirmée aussi bien par les entrepreneurs qui participent à une coentreprise que par ceux qui prévoient le faire. Le diagramme en bâtonnets permet de déduire que les exportations vers les pays du tiers monde sont aussi bien élevées pour les entrepreneurs «pro-coentreprise » que pour ceux « anti-coentreprise », venant confirmer les résultats de Dagevos et De Groot (1990) dans leur enquête dans la province du Brabant aux Pays-Bas. Les importations sont en revanche limitées, ce qui s'explique par le fait que la majorité des PME restreignent leurs importations aux pays voisins. Mais en ce qui concerne les autres activités, on peut constater des différences considérables entre les deux groupes. Les entrepreneurs «pro-coentreprise» travaillent plus en sous-traitance $(\mathrm{p}<0,01)$ et pour une

1. En annexe, nous commentons la technique, mais pour plus de détails techniques, voir Gifi (1981) et van den Berg (1988). 
organisation belge (par exemple, une organisation non gouvernementale) ou étrangère $(\mathrm{p}<0,05)$, accordent plus de licences dans les pays en voie de développement $(\mathrm{p}<0,01)$ et y font produire plus sous leur marque commerciale (franchises, $\mathrm{p}<0,01$ ). Nous relevons également que $10,6 \%$ des entrepreneurs qui ne sont pas intéressés par la coentreprise veulent être propriétaires à $100 \%$ d'une filiale commerciale dans un pays en voie de développement; pour l'unité de production, ce pourcentage est de $6,2 \%$.

\section{GRAPHIQUE 1}

Types de relations avec les pays en voie de développement (plusieurs réponses possibles)

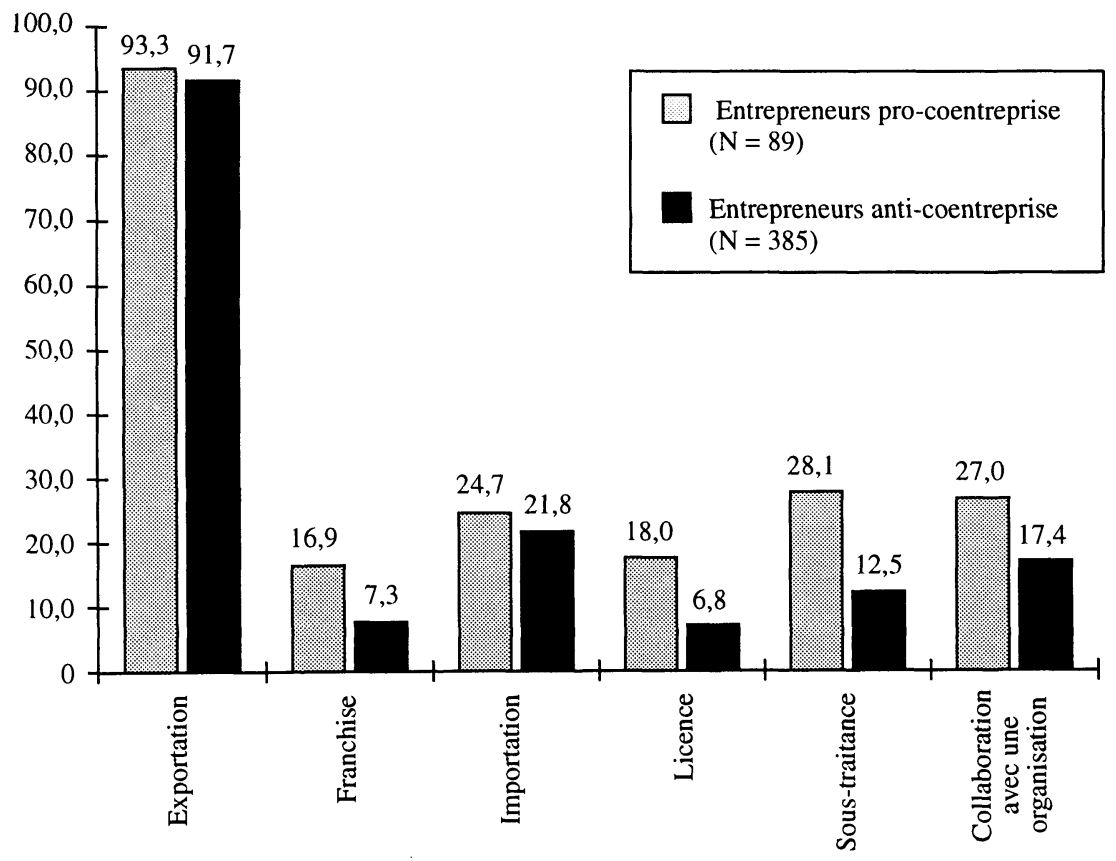

\subsubsection{Travail d'exploration}

Le tableau 1 indique que les entrepreneurs «pro-coentreprise » explorent plus de domaines d'information avant de se lancer dans un pays en voie de développement. Cela est aussi vrai en ce qui concerne l'origine des sources de financement qu'ils ont utilisées $(\mathrm{p}<0,05)$. Environ $60 \%$ des entrepreneurs «pro-coentreprise », contre $46,4 \%$ des autres $(\mathrm{p}<0,05)$, ont en effet financé leurs activités dans les pays en voie de développement autrement que par leurs 
propres moyens. Ils exploitent davantage les possibilités de soutien gouvernemental sur les plans régional, national et européen. Ainsi, les entrepreneurs «pro-coentreprise » se tournent manifestement vers des sources plus diversifiées d'information, comme l'hypothèse 2 le postule.

TABlEAU 1

Travaux d'exploration pour entrer dans un pays en voie de développement

\begin{tabular}{|c|c|c|c|c|}
\hline & & $\begin{array}{c}\text { Entrepreneurs } \\
\text { « pro- } \\
\text { coentreprise " } \\
(\%)\end{array}$ & $\begin{array}{l}\text { Entrepreneurs } \\
\text { qui excluent } \\
\text { une } \\
\text { coentreprise } \\
(\%)\end{array}$ & $\begin{array}{l}\text { Test } \\
\text { du Chi } \\
\text { carré }\end{array}$ \\
\hline $\begin{array}{l}\text { Réalisation d'une étude de marché } \\
(\mathrm{N}=432)\end{array}$ & $\begin{array}{l}\text { Oui } \\
\text { Non }\end{array}$ & $\begin{array}{l}65,5 \\
34,5\end{array}$ & $\begin{array}{l}42,5 \\
57,5\end{array}$ & $\mathrm{p}<0,01$ \\
\hline $\begin{array}{l}\text { Participation à des séminaires } \\
(\mathrm{N}=429)\end{array}$ & $\begin{array}{l}\text { Oui } \\
\text { Non }\end{array}$ & $\begin{array}{l}41,0 \\
59,0\end{array}$ & $\begin{array}{l}22,3 \\
77,7\end{array}$ & $\mathrm{p}<0,01$ \\
\hline $\begin{array}{l}\text { Informations obtenues auprès } \\
\text { d'organismes du pays en voie } \\
\text { de développement }(\mathrm{N}=427)\end{array}$ & $\begin{array}{l}\text { Oui } \\
\text { Non }\end{array}$ & $\begin{array}{l}50,6 \\
49,4\end{array}$ & $\begin{array}{l}27,9 \\
72,1\end{array}$ & $\mathrm{p}<0,01$ \\
\hline $\begin{array}{l}\text { Informations obtenues auprès } \\
\text { d'organismes de l'UE } \\
(\mathrm{N}=423)\end{array}$ & $\begin{array}{l}\text { Oui } \\
\text { Non }\end{array}$ & $\begin{array}{l}30,5 \\
69,5\end{array}$ & $\begin{array}{l}19,4 \\
80,6\end{array}$ & $\mathrm{p}<0,05$ \\
\hline
\end{tabular}

\subsubsection{Les expériences}

Les entrepreneurs « pro-coentreprise » sont nettement plus nombreux à souligner qu'ils ont connu des problèmes lors du démarrage de leurs activités dans un pays en voie de développement $(\mathrm{p}<0,05)$ - environ $81 \%$ des entrepreneurs « pro-coentreprise », contre $69,3 \%$ du groupe « anti-coentreprise »-ce qui confirme l'hypothèse 3 . Par exemple, les problèmes avec la main-d'œuvre locale sont plus fréquents $(20,6 \%$ contre $7,7 \%, \mathrm{p}<0,01)$. Ces différences indiquent qu'une coentreprise dans un pays en voie de développement ne peut pas être réalisée en un tournemain.

En matière de facteurs déterminants pour la réussite dans les pays du tiers monde, le groupe «pro-coentreprise » attache plus d'importance à un plan écrit détaillé ( $\mathrm{p}<0,05$ ), soit environ $62 \%$ contre $47,9 \%$ pour des entrepreneurs qui excluent une coentreprise. Cette constatation contraste avec celle de Ferdows (1980) et réfute, par conséquent, l'hypothèse 4. 
Enfin, un chiffre d'affaires élevé dans les transactions avec les pays en voie de développement est plus fréquent dans le groupe «pro-coentreprise » $(\mathrm{p}<0,01)$. Plus de la moitié des entrepreneurs « pro-coentreprise » $(52,5 \%)$ réalisent au moins $30 \%$ de leur chiffre d'affaires grâce aux exportations vers les pays en voie de développement. Chez les entrepreneurs qui ne sont pas intéressés à la coentreprise, $36,3 \%$ atteignent ce pourcentage de chiffre d'affaires. L'hypothèse 5, selon laquelle les PME qui s'engagent dans une coentreprise dans un pays en voie de développement sont plutôt celles qui y réalisent déjà un bon chiffre d'affaires, est donc confirmée.

Étant donné les résultats très convenables dans un pays en voie de développement, il n'est pas étonnant que la plupart des dirigeants de PME belges interrogés veuillent étendre leurs activités à d'autres pays en voie de développement $(\mathrm{p}<0,05)$. Les entrepreneurs « pro-coentreprise » qui prévoient cette extension sont, toutefois, plus nombreux $(85,2 \%$ contre $73,7 \%)$.

\subsubsection{Caractéristiques propres aux entrepreneurs}

Les entrepreneurs «pro-coentreprise » sont largement plus âgés $(\mathrm{p}<0,05)$ : environ $80 \%$ ont plus de 39 ans contre 68,2\% dans l'autre catégorie. La deuxième différence significative concerne le niveau de scolarité $(\mathrm{p}<0,01)$ : la catégorie « coentreprise » comprend nettement plus d'universitaires $(80,2 \%$ contre $62,4 \%$ ). En ce qui concerne la connaissance des langues, il n'existe pas de différence significative entre les deux groupes $(44,9 \%$ des entrepreneurs «pro-coentreprise » parlent au moins trois langues, contre 40,5\% du groupe « anti-coentreprise »). L'hypothèse 6 , où nous avons supposé que le degré de scolarisation et de connaissance des langues influence la décision de s'engager dans une coentreprise dans un pays en voie de développement, est donc partiellement confirmée.

\subsection{Analyse non linéaire en composantes principales}

L'analyse non linéaire en composantes principales permet d'établir une relation entre les variables décrites ci-dessus qui diffèrent significativement, d'une part, et la possession ou prévision ou non d'une coentreprise dans un pays en voie de développement, d'autre part. Le résultat de cette analyse est présenté au graphique 2, avec le graphique des coordonnées des différentes variables suivantes : 
NLIC et NORG coïncident.

- GMPLA/NBPLA/BPLA = pas d'opinion sur un plan d'affaires écrit et détaillé / trouve le plan pas important / le trouve important.

- NEOL/EOL = pas d'extension / extension à d'autres pays en voie de développement.

- NAFIN/AFIN = financement autre que les propres moyens : pas utilisé / utilisé.

- HKT/HLT = pas de formation universitaire / formation universitaire.

- NMAO/MAO = étude de marché non réalisée / réalisée.

- NIOL/IOL = pas d'informations obtenues auprès d'institutions du pays en voie de développement / informations recueillies.

- -39LFT/40-LFT = entrepreneur âgé de moins de 40 ans / âgé de 40 ans ou plus.

- NIEU/IEU = pas d'informations obtenues auprès des institutions de l'UE / informations obtenues.

- NSEM/SEM = pas de participation / participation à des séminaires.

- NJV/JV = pas de coentreprise / une coentreprise actuellement prévue.

- $-29 \mathrm{AEX} / 30-\mathrm{AEX}=$ moins de $30 \%$ du chiffre d'affaires grâce aux exportations vers les pays en voie de développement / au moins $30 \%$.

- NSUB/SUB = ne travaille pas / travaille en sous-traitance dans les pays en voie de développement.

- NLIC/LIC = n'octroie pas / octroie des licences dans des pays en voie de développement.

- NORG/ORG = ne travaille pas / travaille pour une organisation dans un pays en voie de développement.

- NFRA/FRA = pas de franchises / franchises dans un pays en voie de développement.

- NPPR/PPR = pas de problèmes de personnel / problèmes de personnel dans le pays en voie de développement. 
GraphiQue 2

Analyse de la coentreprise par l'analyse non linéaire en composantes principales

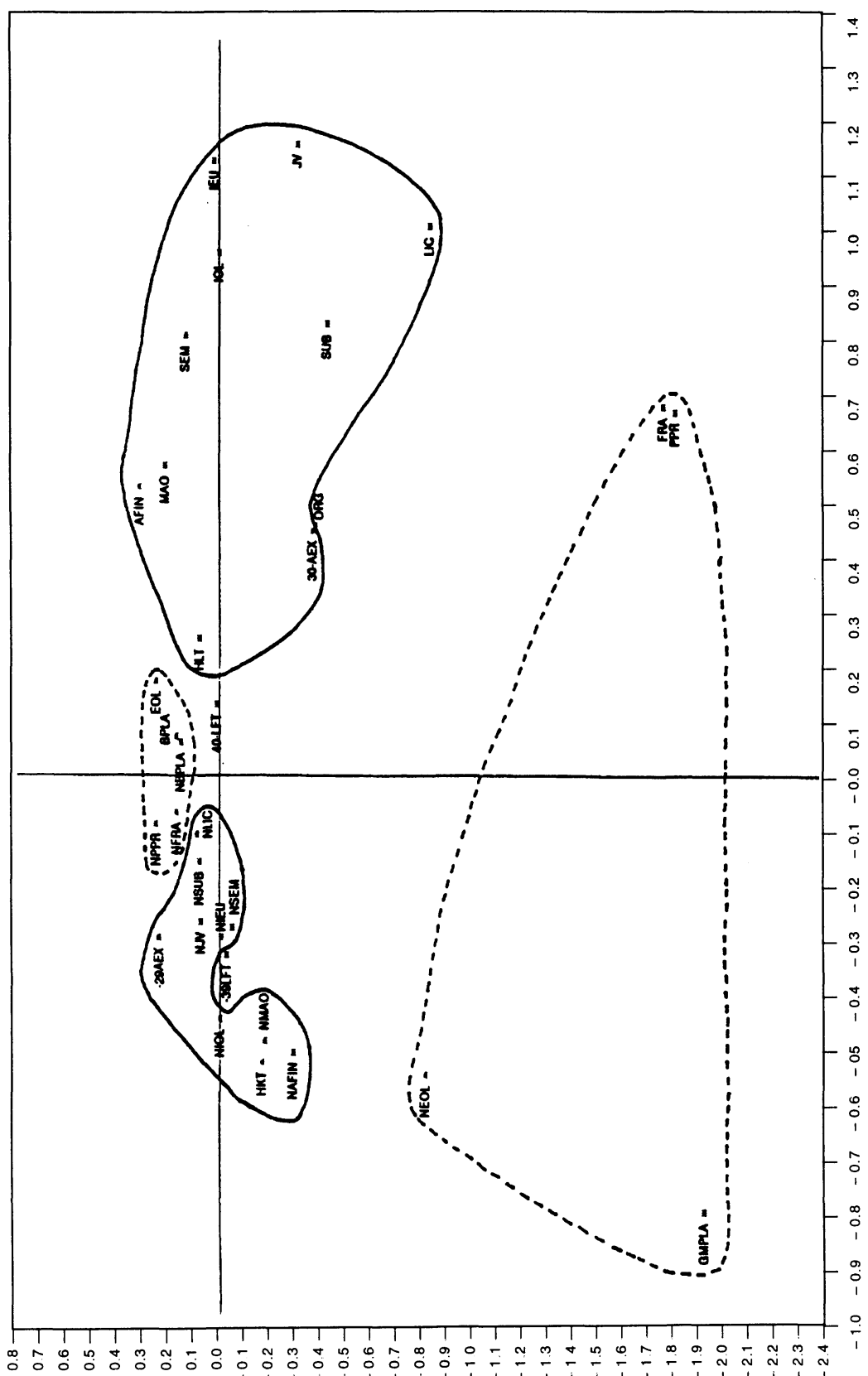

Revue internationale P.M.E., vol. 8, $\mathrm{n}^{\circ}$ 1, 1995 
Les coordonnées des catégories des variables qui ont un poids élevé lié aux composantes principales sur l'axe horizontal (la première composante principale) sont connectées et sont entourées d'une ligne continue. Les coordonnées des variables qui ont des charges élevées liées aux composantes principales sur l'axe vertical (la deuxième composante principale) sont encerclées d'une ligne interrompue. Il existe deux variables (l'âge avec les catégories -39LFT/40-LFT et travailler pour une organisation avec les catégories NORG/ORG) dont les poids liés aux composantes principales sont trop faibles pour qu'on les attribue à l'une des deux composantes principales. Nous étudierons les catégories des variables qui se projettent sur l'axe horizontal, car la variable de la coentreprise (avec les coordonnées de catégorie NJV/JV) appartient à cette dimension.

Nous remarquons un lien étroit entre une position incertaine dans la recherche d'informations et l'absence de coentreprise. Les variables qui se rapportent à la non-recherche d'informations (NMAO, NSEM, NIOL, NIEU et NAFIN) se rapportent en fait au NJV. Par contre, les entrepreneurs " procoentreprise » ont mis en marche des mécanismes d'information. Le rapport entre recherche d'informations et coentreprise est, toutefois, moins fort qu'en cas d'absence de coentreprise, comme le montre l'écart entre les variables de recherche d'informations (MAO, SEM, IOL, IEU et AFIN) et JV. L'hypothèse 2 concernant le besoin d'une recherche d'informations pour mettre sur pied une coentreprise est donc confirmée de façon irréfutable. L'analyse des composantes principales nous apprend, en outre, qu'il existe également un lien étroit entre l'absence d'une coentreprise et d'autres formes d'activités avec les pays en voie de développement (NLIC et NSUB). Enfin, nous constatons qu'un bon chiffre d'affaires favorise la création d'une coentrepris (voir la distance entre JV et 30-AEX et NJV et -29AEX), ce qui confirme l'hypothèse 5.

\section{Conclusion}

Dans cet article, nous avons examiné la coentreprise dans un pays en voie de développement entre PME de pays industrialisés et pays du tiers monde. Ce lien de collaboration offre, en effet, de bonnes perspectives à long terme et présente donc de bonnes chances de stabilité. Cependant, la coentreprise n'est pas a priori l'objectif. Chaque entrepreneur qui développe une activité avec un pays en voie de développement n'envisage pas nécessairement une coentreprise. En outre, cette forme de collaboration est le prolongement d'un lien antérieur de collaboration moins étroite, qui est souvent une relation commerciale. Pendant cette période d'alliance moins étroite, les partenaires voient si leurs comportements convergent et apprennent à connaître la culture de l'autre ; ils améliorent la communication et créent des liens de confiance (Cascio et Seramio, 1991). 
Nous retenons également que les dirigeants de PME du pays développé qui établissent une coentreprise disposent de plus de sources d'information. Ils ont, notamment, davantage recours à l'aide publique, et l'étude de marché, les séminaires, la collecte d'informations auprès de diverses instances, etc., font partie de leur démarche d'informations. Bref, une préparation particulière est nécessaire avant de conclure un lien de collaboration solide avec l'entrepreneur du pays en voie de développement. Les résultats empiriques donnent aussi des indications sur l'importance des infrastructures d'appui. En raison des besoins d'information, il est essentiel que les organismes d'appui tiennent compte du caractère propre des PME. Ils doivent comprendre qu'une PME n'est pas une grande entreprise en miniature et la livraison d'informations aux dirigeants de PME suppose donc du travail sur mesure ; les entrepreneurs désirent rapidement des informations sommaires et pratiques. Enfin, l'enquête a montré que les objectifs de croissance et ceux du développement peuvent coexister. Les responsables de la coopération pour favoriser le développement des pays pauvres doivent aussi promouvoir l'économie et le commerce. Une coentreprise dans un pays en voie de développement, qui sert également les intérêts des entrepreneurs et des PME locales, est, en effet, souvent précédée de liens commerciaux qui se perpétuent par la suite. Les autorités compétentes pour le commerce international doivent de leur côté intégrer les aspects du développement dans leur politique. Ainsi, elles contribueront à la réalisation de diverses formes de collaboration qui créeront un levier de développement pour les pays du tiers monde. 


\section{Annexe}

L'analyse non linéaire en composantes principales permet d'analyser des variables qui se situent à tous les niveaux de mesure (intervalle, ordinal et nominal). Grâce à cette technique, nous pouvons trouver quelles variables sont corrélées entre elles.

Pour cela, une nouvelle variable $x$, soit la composante principale ou la dimension, est calculée comme suit :

$$
x_{i}=\left(a_{1} z_{i 1}+a_{2} z_{i 2}+\ldots+a_{m} z_{i m}\right) / m=\sum_{j=1}^{m} a_{j} z_{i j} / m
$$

- où $i$ indique l'objet observé (ici les entrepreneurs); $m$, le nombre de variables ; $z$, que les variables sont standardisées et $a$, le poids. Ces derniers sont choisis de façon à ce que la nouvelle variable $x$ corresponde le mieux avec chacune des variables originales. Le poids est égal à la corrélation entre la variable et la composante principale, et cette corrélation est le poids lié aux composantes principales. Plus la valeur absolue du poids lié à la composante principale est grande, plus la relation est forte entre la variable et la composante principale : cette variable est donc mieux représentée par cette composante principale. Des variables en forte corrélation présentent, par conséquent, des poids liés à la composante principale qui sont élevés sur la même composante principale. Une variable est supposée appartenir à une composante principale, quand la valeur absolue de son poids lié à la composante principale est au moins la moitié de la valeur absolue du poids lié à la composante principale, qui est la plus élevée sur cette dimension. La détermination du nombre de dimensions dépend de l'interprétation des résultats et des valeurs propres - la moyenne des corrélations carrées entre la composante principale et les variables. Quand la valeur propre est inférieure à $1 / m$, la composante principale n'est pas retenue. Une dimension ne sera donc pas utilisée, si sa valeur propre est nettement inférieure aux précédentes. Nous remarquons également que la corrélation entre les composantes principales doit être égale à 0 . 


\section{Bibliographie}

BEAMISH, P.W. (1987), "Joint ventures in LDC's : partner selection and performance », Management International Review, 1, p. 23-37.

BERLEW, F.K. (1984), « The joint venture - a way into foreign markets », Harvard Business Review, juillet-août, p. 48-51.

BuCKLEY, P.J. et M. CASSON (1988), «A theory of cooperation in international business ", Management International Review, Édition spéciale, p. 19-38.

CASCIO, W.F. et M.G. SERAMIO (1991), « Human resources systems in an international alliance : the undoing of a done deal ? ", Organizational Dynamics, hiver, p. 63-74.

CONTRACTOR, F.J. et P. LORANGE (1988), « Competition vs. cooperation : a benefit / cost framework for choosing between fully-owned investments and cooperative relationships », Management International Review, Édition spéciale, p. 5-18.

Dagevos, J. et G. DE Groot (1990), Brabants bedrijfsleven en ontwikkelingslanden (La vie économique dans la province du Brabant et les pays en voie de développement), Tilburg, Institut pour des questions de développement.

DATTA, D.K. (1988), «International coentreprises : a framework for analysis », Journal of General Management, 2, p. 78-91.

DAWSON, L.M. (1982), « Opportunities for small business in third world markets », American Journal of Small Business, juillet-septembre, p. 19-26.

DONCKELS, R. et G. ELSHOUT (1992), KMO's en hun omgeving, een studie over de netwerkpositie van KMO'ers in Vlaanderen (Des PME et leur environnement, une étude sur la position de réseau des entrepreneurs en Flandre), Bruxelles, BAC, Centre d'études des PME, Université Catholique de Bruxelles.

DONCKELS, R. et R. AERTS (1993), Les PME et l'internationalisation, dans P. Bernard et al. (dir.), Action-export, un guide pratique des aides, Bruxelles, Roularta Books, Chambre de commerce et d'industrie de Bruxelles.

FERDOws, K. (1980), «Start-up of small manufacturing ventures in developing countries », American Journal of Small Business, octobre-décembre, p. 12-22.

GIFI, A. (1981), Nonlinear Multivariate Analysis, Leiden, Department of Data Theory University.

GOMES-CASSERES, B. (1989), "Joint ventures in the face of global competition », Sloan Management Review, 3, p. 17-26.

HAMEL, G. (1991), «Competition for competence and interpartner learning within international strategic alliances », Strategic Management Journal, Édition spéciale, été, p. 83-103. 
HARRIGAN, K.R. (1988), «Joint ventures and competitive strategy », Strategic Management Journal, 2, p. 141-158.

HENNART, J.F. (1988), «A transaction costs theory of equity joint ventures », Strategic Management Journal, 4, p. 361-374.

Kogut, B. (1988), «Joint ventures: theoretical and empirical perspectives », Strategic Management Journal, 4, p. 319-332.

LAMBRECHT, J. (1993), «Cooperation agreements between entrepreneurs from developed and developing countries, theoretical considerations and empirical evidence from Belgium », Southern African Journal for Entrepreneurship and Small Business, 1, p. 12-20.

LEI, D. et J.W. SLOCUM (1991), «Global strategic alliances : payoffs and pittfalls », Organizational Dynamics, hiver, p. 44-62.

NEWMAN, W.H. (1992), “ "Focused coentreprises" in transforming economies », The Executive, 1, p. 67-75.

PASQUeT, P. (1988), «Coopération industrielle - la " joint venture " », Le MOCI, 25 janvier, p. 15-38.

SAmuels, J., S. GReENFIELD et H. MPUKu (1992), « Exporting and the smaller firm », International Small Business Journal, janvier-mars, p. 24-36.

SCHILlACI, C.E. (1987), « Designing successful joint ventures », Journal of Business Strategy, 2, p. 59-63.

SIMIAR, F. (1983), "Major causes of joint venture failures in the Middle East : the case of Iran », Management International Review, 1, p. 58-67.

VAN DEN BERG, G.M. (1988), Princals voor beginners (Princals élémentaire), Leiden, Datatheorie Vakgrœp Rijksuniversiteit Leiden. 